\title{
Endovascular balloon assistance during hip disarticulation
}

\author{
Roberto Azzoni ${ }^{a}$, Giovanni Nano ${ }^{b}$, Ilias Dalainas ${ }^{b}$, Paolo Bianchi ${ }^{b}$ and Silvia Stegher ${ }^{b}$
}

\section{INTRODUCTION}

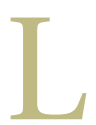

imb disarticulation has been widely performed since the 18th century, especially in war surgery when reconstructive interventions have not been possible. ${ }^{1}$ Actually, limb disarticulation is infrequently done in orthopaedic and vascular surgery, and it is associated with a high mortality rate because of frequent comorbidities. ${ }^{2}$ Hip disarticulation usually is reserved for patients with neoplasms or gangrene from severe artherosclerosis.

During hip disarticulation, hemodynamic stability can be altered by hemorrhagic events in the femoral artery. The authors propose an endovascular technique for proximal control of the femoral artery to reduce blood loss during hip disarticulation. The procedure was done in a 78-year-old man who had a history of prostate resection for cancer and was under oncological treatment at our institution for 10 years. He developed bone and muscle metastases in the left femur with femoral neck involvement. Because of the slow progression of the disease and the absence of other metastatic lesions, life expectancy was considered to be good, and it was decided to proceed with left hip disarticulation.

Because of the proximal location of the tumor, near the femoral neck, the use of a compressive tourniquet was considered impossible, and the vascular surgeon recommended controlling an eventual hemorrhagic event by placing an angioplasty balloon catheter into the left external iliac artery. Color-duplex scanning was performed preoperatively, which showed patency of the iliac and femoral vessels with severe calcifications. Plain abdominal radiographs confirmed diffuse calcification in the arterial axis.

\section{TECHNIQUE}

Surgery was performed using spinal anesthesia. The vascular access was percutaneous at the right common femoral artery. A 6 French (Fr) introducer sheath (Avanti Introducer Sheath, Cordis Johnson \& Johnson, Miami, USA) was placed using the Seldiger technique. Under fluoroscopic control, with a

\footnotetext{
${ }^{a} 1$ st Orthopedic Department, Policlinico San Donato, University of Milan, Italy

${ }^{b} 1$ st Vascular Surgery Department, Policlinico San Donato, University of Milan, Italy

Correspondence to Roberto Azzoni, MD, Orthopedic Department,

Policlinico San Donato, 20095 San Donato Milanese, 30 Morandi Street, Italy

Tel: +00390252774632; fax: +00390252774632;

e-mail: roberto.azzoni@unimi.it

1940-7041 @ 2009 Wolters Kluwer Health | Lippincott Williams \& Wilkins
}

portable vascular C-arm capable of digitally subtracted angiogram and roadmap angiography, a 0.035-in hydrophilic guide wire (Terumo, Leuven, Belgium) was crossed over into the left iliac artery through a 5F contra angiographic catheter (Boston Scientific, Natick, USA) placed at the aortic bifurcation. After a diagnostic angiography (Figure 1) the guide wire was replaced with an Amplatz 0.035-in, 260-cm long, super stiff guide wire (Boston Scientific, Natick, USA). Then, a 7-mm x 20-mm Ultra-thin ${ }^{\mathrm{TM}}$ SDS balloon catheter (Boston Scientific, Natick, USA) was placed in the left external iliac artery (Figure 2), and systemic heparinization with 5000 UI was performed. The balloon catheter was inflated (Figure 3) and femoral pulsation ceased immediately. After proximal, endovascular occlusion, left hip disarticulation was accomplished without any hemorrhagic complication. At the end of the procedure, the balloon was deflated and removed. Hemostasis of the surgical field completed the procedure. The femoral access in the right common femoral artery was controlled with a 6 Fr Angio-seal percutaneous hemostatic system (St Jude Medical, Zaventen, Belgium).

\section{DISCUSSION}

Amputation and disarticulation are infrequent, but sometimes necessary, interventions in orthopaedic surgery. The most frequent indications are major traumatic injuries when reconstruction is not possible, particularly war injuries,

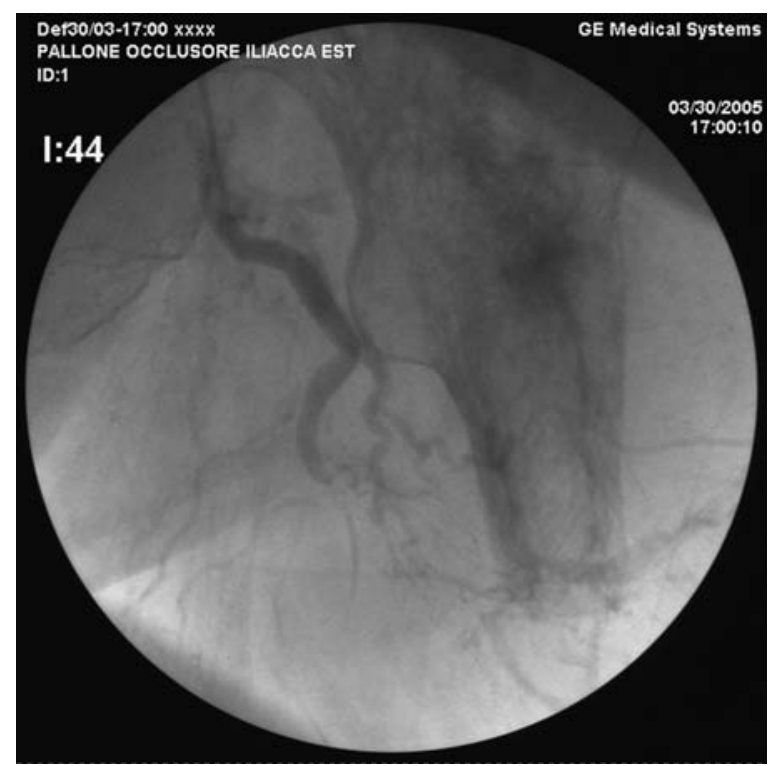

FIGURE 1. Selective angiography of the left iliac axis. 


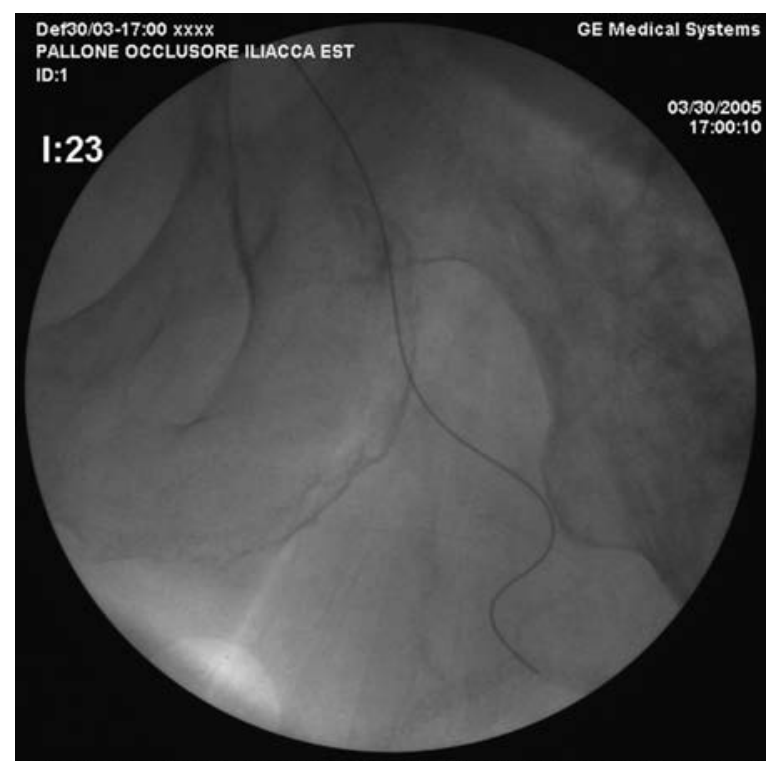

FIGURE 2. Guide wire into the left iliac axis with right femoral access.

radical treatment for primitive neoplastic disease of the bone or soft tissues and irreversible ischemic lesions. In vascular surgery, it is performed in patients in whom reconstructive interventions have failed because of poor outflow and irreversible gangrene. Hip disarticulation is considered to be a radical major intervention; $90 \%$ are done for neoplastic disease. ${ }^{3}$ This type of amputation is still associated with high mortality and morbidity, even with improved anesthesiological management, antibiotics, blood transfusions and surgical techniques.

In hip disarticulation, hemostatic tourniquets cannot be used because of the location of the operating field. Therefore, control of bleeding is a major issue in this procedure, and orthopaedic surgeons should try to avoid hemorrhagic complications with or without the support of vascular procedures. Various techniques have been proposed to reduce blood loss. The first modern technique was proposed by Boyd in $1947^{4}$ in which femoral vessels and nerves were ligated before the disarticulation. Endovascular balloon artery occlusion has been used for initial assessment of ruptured abdominal aortic aneurysms, ${ }^{5,6}$ as well as in laparoscopic surgery in the iliac arterial axis for aneurysmal disease, ${ }^{7}$ but to our knowledge it has not been reported to control bleeding during hip disarticulation.

The use of semi-compliant balloon catheters for endovascular occlusion avoids injury to the endothelium of the vessel wall during balloon inflation. Preoperative assessment, however, with color-duplex scanning and plain abdominal radiographs, is mandatory; coexisting atherosclerosis often

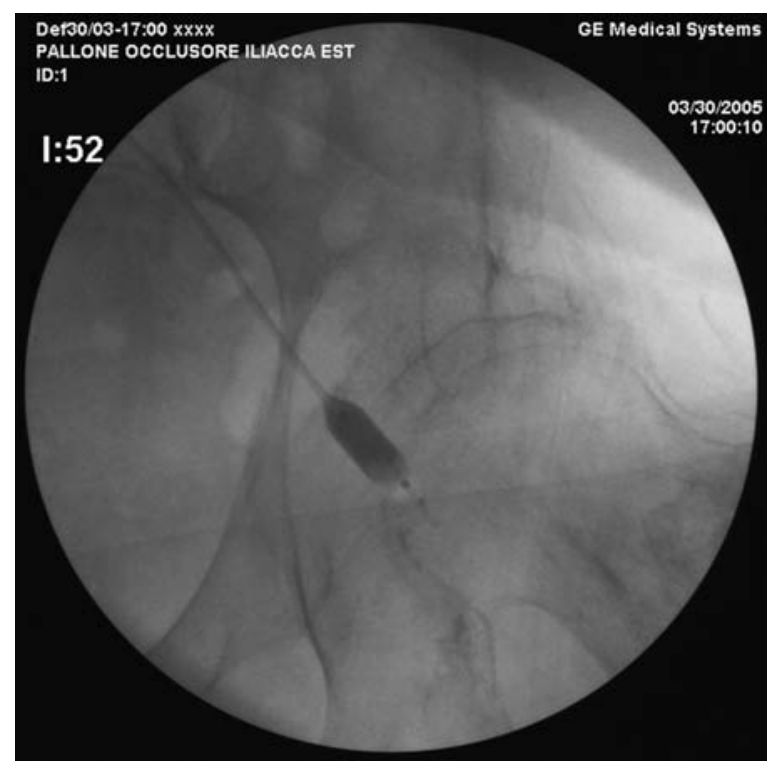

FIGURE 3. Balloon inflation and endovascular occlusion.

is present especially in elderly patients, and severe wall calcification can lead to vessel rupture and retroperitoneal hematoma, or even balloon catheter rupture. Moreover, color-duplex scanning and radiographs will help in choosing the landing-zone for balloon inflation. In hip disarticulations, contralateral femoral artery access is recommended to clear the operating field.

In conclusion, endovascular balloon assistance is a simple, safe and effective in preventing major arterial bleeding during amputation or disarticulations and can be routinely used.

\section{REFERENCES}

1. Hospital Report. Guy's Hospital, January 16. Amputation at the hip joint. Lancet. 1824; 2:96-97.

2. Wakelin SJ, Oliver CW, Kaufman MH. Hip disarticulation - the evolution of a surgical technique. Injury. 2004; 35:299-308.

3. Loon HE. The past and present significance of hip disarticulation. Artif Limbs. 1957; 4:4-21.

4. Boyd HB. Anatomic disarticulation of the hip. Surg Gynecol Obstet. 1947; 84:346-349.

5. Malina M, Veith F, Ivancev K, Sonesson B. Balloon occlusion of the aorta during endovascular repair of ruptured abdominal aortic aneurysm. J Endovasc Ther. 2005; 12:556-559.

6. Dalainas I, Nano G, Bianchi P, et al. Endovascular technique for the treatment of ruptured abdominal aortic aneurysms: 7-year intention-to treat results. World J Surg. 2006; 30:1-6.

7. Wassiliew S, Kolvenbach R, Puerschel A, Schwierz E. Total laparascopic iliac artery aneurysm repair using endoscopic techniques and endovascular balloon occlusion. Eur J Vasc Endovasc Surg. 2006; 32:270-272. 